Meta

Journal des traducteurs

Translators' Journal

\title{
Statistiques de la servitude en matière de traduction
}

\section{Jean-Paul Vinay}

Volume 25, numéro 4, décembre 1980

URI : https://id.erudit.org/iderudit/004567ar

DOI : https://doi.org/10.7202/004567ar

Aller au sommaire du numéro

Éditeur(s)

Les Presses de l'Université de Montréal

ISSN

0026-0452 (imprimé)

1492-1421 (numérique)

Découvrir la revue

Citer cet article

Vinay, J.P. (1980). Statistiques de la servitude en matière de traduction. Meta, 25(4), 447-454. https://doi.org/10.7202/004567ar d'utilisation que vous pouvez consulter en ligne.

https://apropos.erudit.org/fr/usagers/politique-dutilisation/ 


\section{Statistiques de la servitude en matière de traduction}

JEAN-Paul VINAY

Ce titre, qui peut paraitre bizarre au premier abord, m'a été suggéré par une remarque qui m'a échappé(e) lors d'un séminaire que je donnai récemment à UBC; nous parlions, bien entendu, de traduction et $\mathrm{j}$ 'essayais de défendre une de mes idées favorites, à savoir que le traducteur ne dispose, dans son travail, que d'une marge extrêmement faible de liberté d'action. Autrement dit, je suis persuadé, après de longues réflexions sur la traduction, que le traducteur ne peut que rechercher des équivalences, qu'il souffre constamment du poids de plusieurs servitudes, que $\mathbf{M}$. Le Quellec appelait récemment, dans une causerie donnée à l'ATIO, «la tyrannie de la forme».

Il est certain qu'un rédacteur, qui ne part pas d'un texte en langue de départ, a le sentiment d'une certaine liberté d'action. C'est ce qui se passe en ce moment même, alors que je laisse mes doigts mourir sur le clavier de ma fidèle Smith-Corona. Mais, même alors, il existe des servitudes linguistiques très fortes, qui obligent tout écrivain à passer sous les fourches caudines de la stylistique interne, faute de quoi il sera mal compris, on ne sera pas compris du tout. Je sais bien que la stylistique interne n'est pas notre propos aujourd'hui, mais je ne puis m'empêcher de faire remarquer que les écrivains qui veulent secouer le joug des servitudes langagières ne peuvent le faire que dans une très faible mesure : mettre un bonnet rouge aux mots du dictionnaire, c'est très bien, cela fait un bon alexandrin. Mais Victor Hugo, tout en proclamant son désir de changer de crèmerie, n'en restait pas moins traditionnel et partant très compréhensible. Je n'en dirais pas autant de la jeune génération de poètes, aussi bien sur les bords de la Seine que sur les bords du Saint-Laurent qui jettent les bonnets rouges par-dessus les moulins et sont, du moins pour moi, tout à fait incompréhensibles. C'est peut-être là, après tout, l'essence même de la poésie...

Mais l'idéal du traducteur n'est pas d'être incompréhensible - bien que cela lui arrive parfois, si j'en juge par les modes d'emploi bilingues que me fournit mon épicier - pour avoir précisément négligé ou refusé d'obtempérer aux diktats des servitudes linguistiques. Les deux règles d'or du traducteur, inscrites sur le fronton de sa cheminée, doivent être: (a) prendre clairement conscience de l'existence des servitudes, (b) s'habituer à les vaincre par la recherche obstinée d'équivalences dans la langue d'arrivée. C'est là, en somme, le programme de base de tout cours de traduction, qu'on le veuille ou non, à moins qu'on ne préfère traduire à coup de dictionnaire, comme ce fut le cas à 
Victoria pour le fameux Fort Rodd Hill, fameux non pour son passé glorieux, car il n'a jamais tiré un seul coup de canon (ce dont je le félicite), mais bien pour ses traductions. Je ne vous en citerai qu'une, qui se trouve à l'entrée du parc de stationnement. D'un côté, on lit NO EXIT, et de l'autre la traduction NE...QUE SORTIE, ne... que étant précisément l'information donnée par les dictionnaires!

Mais je vous ai laissé en panne avec mon titre: en fait, expliquant aux étudiants de UBC la jungle des servitudes qui risque d'étouffer l'apprentitraducteur, je me suis laissé aller à un jugement statistique. J'ai affirmé, un peu à la légère sans doute, «que $75 \%$ du texte traduit est le résultat de servitudes qui ne laissent au traducteur aucun choix". Après la conférence, un collègue perspicace me demande si cette affirmation était le résultat de sondages véritables, et je ne pus que lui avouer que non, c'était là un sondage au pifomètre, qu'il conviendrait en effet de soumettre à une vérification expérimentale. C'est à cette vérification que je vous convie aujourd'hui: non que nous puissions le faire ici, bien entendu. Mais on peut en parler, et la suggérer à un chercheur en mal de thèse. Je me propose donc de préciser les différentes sortes de servitude qui nous assaillent, pour essayer de voir comment on pourrait en estimer l'importance en termes de pourcentages. Je ne me fais pas d'illusions : c'est là une tâche difficile, peut-être impossible sans un plus haut degré de formalisation des techniques de la traduction. Mais on peut au moins essayer.

\section{DÉFINITION}

Le terme Servitude est utilisé en stylistique comparée [Voir SCFA, § 11] pour rappeler le fait que dans la mesure où la langue nous est donnée, nous sommes contraints de nous y soumettre. Les faits de langue nous sont en effet imposés : genre des mots, conjugaison des verbes, accords des mots entre eux, ordre des mots (le plus souvent); c'est pourquoi les transformationnalistes s'amusent à chercher la preuve de leurs règles en rapprochant des énoncés «licites» et des énoncés «défendus». S'il y a plusieurs énoncés licites, on se trouve alors devant une option, qui représente une toute petite bouffée de liberté langagière: Ex. :

1. La bonne lui a donné une banane.

2. Marie lui a donné une banane.

3. On lui a donné une banane.

* ? 4. Une banane lui a été donnée par la bonne.

*?? 5. Il lui a été donné une banane par la bonne,

et autres variantes du code postal.

Un esprit chagrin, mais peut-être réaliste, pourrait dire alors: «Dans la langue, tout est servitude», et alors ainsi la discussion. Cela ne serait pas tout à fait exact, car je suis libre, après tout, de dire «On lui a donné une banane » ou «Des gens que je ne connais pas lui ont donné une banane». Le message serait le même, en gros. De même, sur une porte de mon ancien lycée, on pouvait lire «Réservé aux Professeurs»; l'année suivante, l'inscription avait été changée 
en «Interdit aux Élèves». Le message était le même, et je n'ai jamais su ce qu'il $\mathrm{y}$ avait derrière cette porte...

Voyons maintenant rapidement quelques-unes de ces fameuses servitudes.

\section{LES SERVITUDES DE LA FORME}

On peut appeler ainsi l'attirance, parfois irrésistible, qu'exercent sur un traducteur les formes extérieures du texte. Par exemple, une construction nominale anglaise appelle une construction nominale française; une locution verbale appelle une locution verbale, etc. Citons quelques faits :

a) L'anglais FROM, en tant que marque d'origine, tend à imposer la traduction $\mathrm{DE}$, bien que le français ait spontanément créé $\mathrm{De}$ la part de, ou (sur les enveloppes) Expéditeur, abrégé le plus souvent en Exp ${ }^{r}$. Et cette servitude est renforcée par la diffusion d'enveloppes imprimées portant la mention FROM/DE. b) L'anglais YIELD a donné naissance à de multiples panneaux CÉDEZ, alors que la rédaction spontanée tend vers la formule PRIORITÉ/À GAUCHE, À DROITE.

c) Les timbres canadiens comportent la mention POSTAGE qui appelle une traduction POSTES ; celle-ci n'est pas absolument nécessaire, comme on peut le voir en examinant des timbres français.

d) La servitude de la forme peut s'appliquer aux espaces graphiques. Par exemple, dans le rapport, pourtant excellent, du Commissaire aux langues officielles (Édition de 1979), on constate l'emploi de PARTIE I, PARTIE II, PARTIE III, correspondant à l'anglais PART I, PART II, PART III.

e) Je ne parlerai que pour mémoire de la servitude de la forme qui aboutit à des traductions ridicules (ce ridicule devrait suffir pour mettre la puce à l'oreille du traducteur), telles que SPELLING BEE, abeille orthographique; PICK-UP TRUCK, camion muni d'un pick-up, etc. On a beau jeu de se moquer de ces simili-traductions; elles n'en existent pas moins, et sont souvent à la base des calques.

f) Le calque est en somme une traduction littérale, généralement correcte (mais pas toujours) sur le plan de la syntaxe, et qui témoigne d'une ignorance de la langue d'arrivée: WHAT'S THE BIG IDEA? Quelle est la grosse idée? On m'objectera que c'est là un exemple grossier, et peu probable; mais j'ai entendu au Québec assez souvent: "Une bonne main pour M. X.» qui serait totalement incompréhensible à qui ne connaîtrait pas l'anglais... Et l'un de mes calques favoris, - favoris par l'indignation qu'il éveille en moi, est l'expression «Si et seulement si...» dans les textes modernes scientifiques (?) pour rendre la locution anglaise IF AND ONLY IF..., alors que quand j'étais potache, il fallait dire, et en fait on disait, et même on ne songeait pas à dire autrement que : «La condition nécessaire et suffisante pour...»"

g) L'exemple le plus extrême de la servitude de la forme est l'emprunt, sorte de démission intellectuelle, parfois justifiée sur le plan technique, le plus souvent résultat d'un snobisme et d'un laisser-aller déplorables. Je sais bien que la masse des usagers se moque bien de ces critiques, et que les calques passent allègrement la rampe, tout au moins dans le français d'Europe; il n'importe, on peut 
toujours relever les emprunts, souvent appelés anglicismes (mais en fait on emprunte de n'importe quelle langue, pourvu qu'elle ait du prestige). Par exemple, je lisais l'autre jour un livre d'Étienne Got, la Pensée binaire, et très vite j'ai perdu de vue le sens profond de cette binarité pour m'arrêter à des phrases telles que celles-ci : "L'idéal est d'arriver... à un rapport tournant autour de la moyenne, c'est-à-dire le fifty-fifty. » Ou encore: "On voit d'ailleurs une confirmation de la théorie de la bisexualité dans le timing de l'action des hormones.» Et naturellement, pas d'italiques, pas de guillemets... Et même la Revue des Lettres, organe de la Société des Gens de Lettres (pas moinsss) nous présente en caractères égyptiens, point 12 , un PLANNING DE LA RÉPARTITION DES DROITS D'AUTEUR. Il doit y avoir aussi un planning des copyrights, je suppose...

Tout de même, «servitude de la forme » est une formule un peu trop globale, trop vague. Essayons de voir rapidement quelles sont les servitudes qui s'imposent à nous sur différents plans du message.

\section{LE PLAN LEXICAL}

Le rapprochement de deux langues à l'intérieur d'un bon dictionnaire bilingue ou par le truchement de deux dictionnaires monolingues permet de trouver, dans les meilleurs cas, des équivalents lexicologiques de langue à langue. Les bons dictionnaires on fait en principe ce travail pour vous, mais pas toujours. Si je cherche reed dans un récent dictionnaire bilingue, je trouve roseau; [musique] anche. Mais les anches sont faites avec des rosseux qui se nomment en anglais cane et non reed, ce que le dictionnaire en question ne dit pas. Cette anche peut, dans certains cas, se fixer à un tube recourbé appelé bocal, mais je ne trouve pas ce terme dans ce même dictionnaire (en anglais, crook, ou parfois bocal), etc.

Il y a plus deux termes peuvent être donnés par le dictionnaire comme étant synonymes, alors qu'en fait ils n'ont pas la même distribution. J'ai été frappé récemment, passant souvent d'un missel français à un missel anglais, par la spécialisation de certains verbes fréquents auxquels je n'avais jamais songé auparavant. Par exemple: Donnez-nous aujourd'hui notre pain quotidien se dit bien Give us our daily bread; mais Donnez-nous la paix se dit Grant us peace. En fait, givelgrant recouvrent presque toujours un donner du français; mais le traducteur n'a pas le choix, comme le dictionnaire semble le faire croire, pas plus qu'il n'a le choix entre in et into pour traduire le seul terme français dans.

On pourrait peut-être chercher une mesure de la servitude dans l'emploi de paires monovalentes, $\mathrm{du}$ type enveloppelenvelope, (postage) stamp/timbre (-poste), key, clef; c'est-à-dire que la traduction de key par clef serait considérée comme le degré zéro d'une gamme de possibilités, telles que [musique, piano] touche; [musique, instrument à vent] clef; [manuel scolaire] corrigé (Canada : solutionnaire), qui constitueraient les degrés de la servitude (dans ce cas, celle du contexte sémantique). Le plus haut degré de la servitude lexicale serait atteint par des énoncés dont le contexte linguistique ne permet pas de décider du 
choix de la bonne traduction, mais seulement le contexte événementiel (ex. : Tear along this line, Close before striking, Ring for the janitor, etc.)

\section{LE PLAN TECHNOLOGIQUE}

Le traducteur peut se trouver ici devant deux situations bien différentes: ou bien la technologie en question existe aussi dans la langue d'arrivée, et c'est alors une servitude. Ou bien elle n'existe pas, et le traducteur doit se faire néologue, inventer des termes, emprunter le cas échéant. Mais ce n'est là que l'illusion de la liberté, car toute néologie crée une servitude pour les générations à venir; tout au plus pourrait-on parler «d'école». Ainsi, pour reprendre l'expression langue d'arrivée (et son contraire, langue de départ) J. Darbelnet et moi-même ont/avons créé ces termes vers 1960 pour rendre Source language et Target language, et maints auteurs les emploient maintenant à titre de servitude - et je les en félicite! D'autres auteurs, par ignorance, par littéralisme, par esprit de contradiction, ont calqué les termes anglais et parlent de "langue source» et «langue cible». Les deux écoles obéissent en fait à deux servitudes : la seule liberté qui vous reste est de mettre, ou d'omettre, les traits d'union...

\section{LE PLAN SYNTAGMatique}

Je grouperais volontiers sous ce chapeau les syntagmes qui reflètent une particularité conceptuelle plutôt que structurale. Par conséquent, je ne parlerai pas de l'emploi des prépositions (s'intéresser àlto be interested in), mais bien des locutions figées qui sont certainement de bons exemples de servitudes. Par exemple, il n'est pas courant dans ma culture particulière d'évoquer un chapeau pour rendre To speak through one's hat ou At the drop of a hat ; inversement, il existe des quantités de chapeaux qui me paraissent incurablement français, tels les chapeaux de roue, les chapeaux chinois, les chapeaux d'un article de journal, etc.

Mais on pourrait m'objecter que ces exemples sont trop exclusivement terminologiques. Prenons alors des exemples de locutions relevées dans les pages vraiment excellentes du Rapport annuel 1979 présenté par le Commissaire aux langues officielles :

...«On souhaite pour le bilinguisme un enterrement sans fleurs ni couronnes» / «One would rather see the whole problem swept under the rug ».

La prime au bilinguisme: Passe-moi la casse, je te passerai le séné

Bilingualism Bonus: To Him that Franc-fief ou franc-parler Hath... On Speaking Terms

L'intérêt de ces rapprochements (que l'on pourrait multiplier, cette édition bilingue s'y prête admirablement) réside dans le fait que les locutions en cause ne sont pas véritablement figées, c'est-à-dire du type filer à l'an- 
glaise / to take French leave. Ce sont en fait des cadres privilégiés pour un certain type de déroulement stylistique, et la recherche des équivalences est d'autant plus délicate qu'elles ne sont pas répertoriées. Ici, la liberté est toute entière dans la chasse à l'équivalence, et seul un traducteur expérimenté peut sentir la pertinence de ces rapprochements. Disons qu'on passe toute sa vie à rechercher des équivalences : ce n'est que tout récemment que j'ai découvert que «Nice work if you can get it» est l'équivalent d'une locution hexagonale qui n'existait pas dans mon enfance : «(Il) faut le faire!»

\section{LE PLAN STYListiQue}

Dans toute langue, il existe des servitudes stylistiques qui traduisent le niveau particulier où se place le discours considéré. Je me promenais l'autre jour dans les allées des Butchart Gardens, célèbres pour leurs magnifiques parterres et leur absence de traduction. Il y avait là un robinet qui coulait, et il faisait chaud. On eût été tenté de boire, sans un écriteau comminatoire : THIS WATER IS UNFIT FOR DRINKING. Par une vieille habitude, j'ai tout de suite réfléchi à sa traduction. Or, il y en a au moins deux, selon le niveau stylistique considéré : Sur le plan officiel, impersonnel, administratif, il faudrait sans doute traduire tout simplement par EAU NON POTABLE, alors que dans un récit de voyage, décrivant un cours d'eau, il faudrait traduire Cette eau n'est pas bonne à boire. On pourrait épiloguer longuement sur cet exemple : remarquons simplement que la liberté de choix entre ces deux traductions n'est qu'apparente, qu'elle relève en fait d'une servitude, celle (particulièrement lourde en français) des niveaux stylistiques. La liberté, si liberté il y a, serait peut-être à chercher dans la rédaction de traductions parallèles, telles que DANGER: $E A U N O N$ POTABLE - EAU CONTAMINÉE - RÉSERVÉ AUX JARDINIERS - NE LAISSEZ PAS LES ENFANTS BOIRE DE CETTE EAU, etc.

\section{LE PLAN CONCEPTUEL/CULTUREL}

Sujet très vaste, et qui a fait couler beaucou d'encre : la langue est le reflet d'une culture et par là même, s'interpose comme un écran entre le texte de départ et le texte d'arrivée. Je n'insisterai pas sur cet aspect essentiel qui colore tout essai de bilinguisme, et dont J. Darbelnet et moi avons donné de nombreux exemples [Notamment toute la partie de la SCFA qui traite des modulations $\S 75-76,216-229$, les paragraphes $246 \mathrm{sqq}$ sur la métalinguistique (terme vieilli maintenant), les allusions $\$ 230 \mathrm{sqq}$, la compensation, $\$ 171 \mathrm{sqq}$, etc.] La recherche des équivalences à ce niveau est difficile, et comme pour les termes techniques, il faut parfois inventer. Mais ce procédé (l'invention) sonne généralement faux - notons qu'on accepte plus facilement les nouveautés de la part d'un auteur que de la part d'un traducteur. Prenons deux exemples : 1'anglais a souvent recours à une locution choquante pour un francophone: Their house is so clean you could eat off the floor. Dans ma culture, on ne mange jamais sur le plancher, peut-être précisément parce qu'il est parfois sale, mais surtout parce que la notion de table (de salle à manger) joue en français un rôle culturel im- 
portant: à table! passer à table; être à table; recevoir quelqu'un à sa table; se lever de table; eau, vin de table; le gite et la table; faire bonne table (bonne chère), etc. Je ne conçois pas un auteur français écrivant: "La pièce était si propre qu'on eût pu manger sur le plancher.» La servitude ici ne repose pas sur le lexique ou la syntaxe, mais sur un aspect conceptuel/culturel que traduit précisément l'abondance des locutions à base de table.

Un deuxième exemple: Mes collègues anglophones étaient friands d'une locution biblique: (They see, They don't see) the writing on the wall. Elle ne me paraît pas usitée en français. On dit bien «Mané, Thécel, Pharès» (cf. Daniel, 25), mais ce n'est pas d'un emploi courant. Que pourrait-on proposer comme équivalent? Je suis certain que mes auditeurs trouveront la solution de ce petit problème, et ne confondront pas cette servitude biblique avec des graffiti ou autres inscriptions officielles. Notons en passant une autre servitude : le dictionnaire donne bien graffiti, mais uniquement au pluriel; que doit-on dire au singulier? Les servitudes culturelles/conceptuelles sont certainement les plus onéreuses, et les plus délicates à dénombrer statistiquement.

\section{UNE BRÈCHE DANS LE MUR DES SERVITUDES?}

J'ai retrouvé des traces d'une certaine liberté en relisant dernièrement la traduction assez récente de Mgr Ronald Knox (The New Testament of our Lord and Serviour Jesus Christ, A New Translation, New York, Sheed and Ward, 1947) et celle de James Moffatt ( $A$ New Translation of the Bible, London, Hodder and Stoughton, 1913-1935). Je comparais dans mon esprit la nouvelle traduction et les anciennes formules, devenues parties intégrantes de la phraséologie de l'anglais, et je voyais là un effet de l'indépendance du traducteur, dans la mesure où les nouvelles traductions ne corrigent pas un original mal compris.

Ai-je raison de croire que ces traductions parallèles, mais qui s'entendent pour diverger, reflètent une certaine marge de manœuvre? J. Darbelnet et moi avions à cet égard introduit dans la terminologie de la stylistique comparée deux notions qu'il n'est peut-être pas inutile de rappeler succinctement. La première est la notion de marge (SCFA, \$ 178): «Il faut donc admettre, écrivions-nous vers 1960 , que le traducteur a une certaine marge de liberté qui n'affecte pas le sens du message, et qui sera d'ailleurs faible dans la mesure où l'analyse des unités de traduction a été soigneusement conduite; d'autant plus que cette marge peut, jusqu'à un certain point, refléter les préférences personnelles du traducteur, son entourage culturel et géographique, - un texte canadien pouvant différer d'un même texte français ou belge par le choix de telle ou telle variante, de synonymes, de tournures locales qui n'influencent pas le sens global du message.»

Nous ajoutions qu'il faut distinguer entre marge et divergence, cette dernière notion étant résolument diachronique, de sorte que, dans l'exemple cité plus haut, on peut accorder à MM. Knox et Moffatt une marge de liberté (synchronique), tout en constatant, en le regrettant parfois, une divergence (dis- 
chronique) entre leurs versions et celles, plus familières, de Douai et de King James.

\section{CONCLUSION}

Terminer n'est pas conclure. Un exposé aussi bref ne saurait prétendre épuiser la question qui, rappelons-le, est de savoir s'il est possible d'envisager une méthode statistique permettant d'évaluer l'importance de la marge (ou inversement l'importance des servitudes) en matière de traduction. Je pose le problème et $\mathrm{j}$ 'attends la réponse. La recherche d'un tel algorithme ne peut être que bénéficiaire (dans le sens où l'on parle de marge bénéficiaire), particulièrement pour reconnaître le poids des servitudes langagières. Comme le disait le professeur Jean Delisle, d'Ottawa, dans le dernier numéro de The $A T A$ Chronicle... «The translator's bilingualism has still another inherent feature: the ability to keep the contrasted linguistic structures intact. It is through his skill in dissociating the two languages that the translator reveals his mastery of the profession.» 